\title{
Gradient Echo MRI
}

National Cancer Institute

\section{Source}

National Cancer Institute. Gradient Echo MRI. NCI Thesaurus. Code C154542.

An application of magnetic resonance imaging that uses spin refocusing and spin echo generation, resulting in shorter repetition times and faster imaging. 